\title{
Caractères physico-chimiques et organoleptiques comparés de jujubes sauvages et des fruits de la variété Gola introduite au Sénégal
}

Pascal Danthu ${ }^{a, b *}$, Pierre Soloviev ${ }^{c, d}$, Anne Tottéc,e ${ }^{c}$ Emmanuel Tine ${ }^{e}$, Nicolas Ayessouf ${ }^{f}$ Abibou Gaye ${ }^{b}$, Thierno Daouda Niang ${ }^{d}$, Moussa Seck ${ }^{b}$, Moussou Falle

\author{
a Cirad-Forêt, \\ BP 1716, Dakar, \\ Sénégal \\ Nouvelle adresse : \\ Cirad-Forêt, \\ BP 853, Antananarivo, \\ Madagascar \\ b Isra/CNRF, \\ BP 2312, Dakar, \\ Sénégal
}

c APEFE Wallonie-Bruxelles, BP 6279, Dakar,

Sénégal

d $\mathrm{CFPH}$,

BP 3284, Dakar,

Sénégal

e LMAGI,

École supérieure Polytechnique, Ucad, BP 5085, Dakar Fann,

Sénégal

${ }^{f} \mathrm{LAE}$,

École supérieure Polytechnique, Ucad, BP 5085, Dakar Fann, Sénégal

pascal.danthu@cirad.fr

* Correspondance et tirés à part

Reçu le 26 juin 2001

Accepté le 17 décembre 2001

Fruits, 2002, vol. 57, p. 173-182 (C) 2002 Cirad/EDP Sciences All rights reserved

DOI: 10.1051/fruits:2002016

RESUMEN Español, p. 182
Jujube trees in Senegal: a comparison between the organoleptic and physicochemical characteristics of the wild fruits and the introduced Gola variety.

Abstract - Introduction. Zizyphus mauritiana Lam. is a wild fruit tree which is widespread in the Sahel region of Africa. Its fruits, jujubes, are traditionally harvested from wild stands and make up a sizeable part of the income of many rural households. They are eaten either fresh or dried. For a few years now, a programme has been under way to introduce improved varieties of jujube trees of Indian origin to Senegal. The present work aimed to compare the fruits of 'Gola', the first introduced variety, with two samples of local jujubes. Material and methods. The comparison concentrated on the biometrical characteristics of the fruits as well as the organic and mineral content of its edible part. This was associated with a tasting session with the consumers. Results and discussion. The edible part in the fresh fruit of the Gola variety is approximately 20 times bigger (volume and weight) than the local fruit. The pulp of the Gola fruit proves to have a higher water content and a lower concentration in organic elements (sugar, protein) and minerals (calcium, magnesium). However, once the fruit is dried, the content does not differ significantly except for protein, and a few minerals in Gola fruit, which are noticeably diminished during the drying process. Dried Gola fruit, very flavorful, is the most highly appreciated by the Senegalese population. Conclusion. Jujubes have a high content of sugar, vitamin $\mathrm{C}$, calcium and iron. These fruits represent an important nutritional contribution for populations of arid zones. The Gola variety suits the consumers' tastes. Its propagation through the Sahel zone is thus a viable option once the technical difficulties linked with the production of the plant are resolved and the economic viability of this new crop is verified.

Senegal / Sahel / Zizyphus ma uritiana / jujube / fruits / chemical composition / food consumption / domestication

\section{Caractères physico-chimiques et organoleptiques comparés de jujubes sauvages et des fruits de la variété Gola introduite au Sénégal.}

Résumé - Introduction. Zizyphus mauritiana Lam. est un arbre fruitier sauvage très commun en Afrique sahélienne. Ses fruits, les jujubes, sont traditionnellement exploités par cueillette dans les peuplements naturels et représentent une source de revenu importante pour de nombreuses familles rurales. Ils sont consommés frais ou secs. Au Sénégal, un programme d'introduction de variétés de jujubiers améliorés d'origine indienne est en cours depuis plusieurs années. L'objectif des travaux présentés a été de comparer la première variété introduite (Gola) avec deux lots de jujubes locaux. Matériel et méthodes. La comparaison entre différentes origines a porté sur les caractéristiques biométriques des fruits et sur les teneurs de la portion comestible en divers composés organiques et en éléments minéraux. L'étude a été complétée par une enquête gustative auprès de consommateurs. Résultats et discussion. La portion comestible des fruits frais de Gola est environ vingt fois plus importante (en poids et volume) que celle des jujubes locaux. La pulpe des Gola est plus riche en eau et moins concentrée en éléments organiques (sucres, protéines) et minéraux (calcium, magnésium). Cependant, après séchage, les teneurs ne sont plus significativement différentes, si ce n'est celles des protéines et de quelques éléments minéraux dont la richesse chez Gola diminue très nettement pendant le séchage. Les fruits de Gola séchés, très parfumés, ont été les plus appréciés par la population sénégalaise. Conclusion. Quelle que soit leur origine, les jujubes sont riches en sucres, en vitamines C, en calcium et en fer. Ces fruits représentent un apport nutritionnel important pour les populations des régions arides. La variété Gola répond bien au goût du consommateur sénégalais. Sa diffusion en zone sahélienne peut donc être envisagée sous réserve d'avoir levé au préalable les contraintes techniques liées à la production des plants et d'avoir vérifié la rentabilité économique de cette nouvelle culture.

Sénégal / Sahel / Zizyphus ma uritiana / jujube / fruits / composition chimique / consommation alimentaire / domestication 


\section{Introduction}

Le jujubier (Zizyphus mauritiana Lam., famille des Rhamnacées) est une espèce ligneuse très commune dans les zones sèches d'Afrique et du sous-continent indien. Cet arbuste a de très nombreux usages, mais son principal intérêt réside dans sa production fruitière [1-3]. Ses fruits, les jujubes, sont des drupes sub-sphériques dont le diamètre est généralement compris entre (1 et 2) $\mathrm{cm}$, à mésocarpe charnu (pulpe) et endocarpe (noyau) unique, dur et lignifié. Les jujubes sont généralement consommés frais ou séchés, mais ils peuvent également être confits, transformés en confiture, en farine ou en jus [3-6].

En Afrique, le jujubier est une essence sauvage dont les fruits sont traditionnellement exploités par cueillette dans les peuplements naturels [7, 8]. En revanche, en Inde et au Pakistan, un très ancien programme d'amélioration a abouti à la création de nombreuses variétés de jujubiers à haut potentiel fruitier [3-5, 9]. Le volume des fruits produits a été multiplié par vingt ou trente par rapport aux jujubes sauvages et un ensemble de techniques culturales spécifiques a été mis au point $[4,5]$. Parmi les variétés indiennes, certaines sont bien adaptées aux conditions arides ; c'est le cas de 'Gola Gurgeon', 'Seb', 'Mundia' ou 'Gola' qui sont des variétés précoces ou bien encore 'Umran' qui est plus tardive [5, 10].

À partir de ces constats, de nombreux auteurs $[2,4,5,11]$ ont envisagé la possibilité d'introduire ces variétés améliorées de Z. mauritiana en Afrique sahélienne. Au Sénégal, ce processus a été engagé depuis cinq ans et il a essentiellement concerné la variété Gola ; les premières récoltes expérimentales ont été obtenues en 1999 [11, 12].

Les travaux présentés ont eu pour objet de comparer les fruits de la variété Gola récoltés au Sénégal et ceux produits par des jujubiers locaux. Pour cela, (i) leurs caractéristiques biométriques, (ii) leur valeur nutritionnelle et (iii) leur qualité organoleptique ont été étudiées.

\section{Matériel et méthodes}

Trois lots de jujubes ont été comparativement étudiés : deux lots ont été récoltés sur des jujubiers autochtones, un troisième l'a été sur la variété Gola introduite et cultivée au Sénégal.

Parmi les jujubiers autochtones, le premier échantillonnage (lot Sindia) a été fait dans une jujuberaie naturelle située près $d u$ village de Sindia $\left(14^{\circ} 35^{\prime} \mathrm{N}, 17^{\circ} 03^{\prime} \mathrm{W}\right)$; les fruits ont été récoltés sur des sujets toutvenant, n'ayant fait l'objet d'aucune sélection préalable. Le deuxième échantillonnage (lot Bandia) a été effectué dans la station expérimentale de Bandia $\left(14^{\circ} 34^{\prime} \mathrm{N}\right.$, $17^{\circ} 01^{\prime}$ W) sur un sujet de 5 ans, identifié 'B13-D16' sur la parcelle et sélectionné sur le critère phénotypique de dimension de ses fruits, supérieure à la moyenne des sujets présents dans la zone récoltée. Le troisième lot de fruits (lot Gola) a été récolté dans un verger expérimental constitué de jujubiers de 2 ans de la variété indienne Gola et localisé à Cambérène $\left(14^{\circ} 45^{\prime} \mathrm{N}, 17^{\circ}\right.$ $\left.26^{\prime} \mathrm{W}\right)$.

Une première série d'analyses a été réalisée sur les jujubes dès leur récolte (fruits frais). Une seconde série de mesures a porté sur des fruits séchés en étuve à $50{ }^{\circ} \mathrm{C}$ pendant $24 \mathrm{~h}$ pour les fruits sauvages et $60 \mathrm{~h}$ pour la variété Gola [6, 13]. La teneur en eau des fruits frais et la teneur en eau résiduelle des jujubes séchés ont été déterminées après chauffage à $105{ }^{\circ} \mathrm{C}[13]$; elles ont été exprimées par rapport à la masse de matière fraîche.

Les teneurs en composés organiques et en éléments minéraux de chaque type de fruits frais ou séchés ont été exprimées pour $100 \mathrm{~g}$ de matière comestible correspondant à la matière fraîche (mf) des fruits frais ou des fruits séchés [7] et pour $100 \mathrm{~g}$ de matière sèche (ms) [13].

Le dosage des protéines a été réalisé selon la méthode de Kjeldhal [14]. La vitamine $\mathrm{C}$ a été dosée par chromatographie en phase liquide et lecture sous UV (200 nm), la phase mobile étant constituée d'un mélange de méthanol, d'hexane sulfonate et de triéthylamine, à $\mathrm{pH} 2,8$. Le dosage des lipides a été réalisé par pesée des matières 
grasses après extraction au Soxhlet par l'éther diéthylique. Les sucres réducteurs totaux et les sucres solubles totaux ont été respectivement dosés par les méthodes colorimétriques de Miller [15] (réaction avec l'acide 3,5 dinitro-salicylique et spectrophotométrie à $550 \mathrm{~nm}$ ) et de Dubois et Gillet [16] (réaction à l'ortho-toluidine et lecture à $630 \mathrm{~nm}$ ). Les résultats ont été exprimés en gramme d'équivalent glucose pour $100 \mathrm{~g}$ de $\mathrm{mf}$ ou ms. L'acidité de la partie consommable a été titrée par une solution de soude $0,1 \mathrm{~N}$ en présence de phénolphtaléine. Elle est exprimée en gramme d'équivalent acide malique pour $100 \mathrm{~g}$ de mf ou ms (indice de correction : 0,067). La quantité de matière minérale totale a été déterminée après incinération à $550^{\circ} \mathrm{C}$ pendant $12 \mathrm{~h}$. Après reprise dans un mélange d'acides chlorhydrique et nitrique, la teneur en phosphore a été évaluée par dosage colorimétrique au réactif vanadomolybdique et lecture à $430 \mathrm{~nm}$. Le fer, le magnésium et le calcium ont été dosés par spectrophotométrie d'absorption atomique.

Les tests gustatifs ont été réalisés sur un panel de 45 personnes choisies au hasard dans divers quartiers de Dakar (Parcelles Assainies, Ouakam, Guédiawaye). L'échantillon a été composé à parts égales de femmes, d'hommes et d'enfants. Chacun des testeurs s'est vu proposé une dégustation de fruits frais et de fruits secs provenant de chacun des trois lots de jujubes préalablement définis. La note de dégustation à attribuer allait de ' 0 ', correspondant aux fruits les moins bons, à ' 5 ' allouée aux meilleurs jujubes.

L'hypothèse d'égalité des moyennes a été testée par analyse de variance. Si elle a été rejetée, les moyennes ont été comparées par la méthode de Newman et Keuls au seuil $\mathrm{P}<5 \%$.

\section{Résultats et discussion}

\subsection{Caractéristiques biométriques des fruits}

Les caractéristiques biométriques des jujubes analysés ont montré que les fruits de la variété Gola avaient un poids et un volume de (9 à 17) fois supérieur à ceux des plants de provenances locales (tableau I). Leur noyau représente moins de $7 \%$ du poids du fruit, alors que, pour les origines Sindia et Bandia, ce rapport a été d'un tiers. De ce fait, le poids et le volume de pulpe consommable chez Gola sont (10 à 20) fois supérieurs à ceux mesurés dans les deux lots de fruits provenant de jujubiers locaux. Le poids moyen des fruits de Gola récoltés au Sénégal $(18,5 \mathrm{~g})$ se révèle cependant un peu inférieur à celui obtenu en Inde : (20 à 24) $g[10,17]$. Cette différence pourrait s'expliquer par des différences environnementales (climat, sol), mais aussi par le fait que les fruits ont été récoltés sur des sujets encore jeunes (première année de fructification) et non taillés ; il a été en effet démontré que la taille modérée des jujubiers permet généralement d'augmenter la biomasse des fruits [17].

Après séchage, le poids de la partie consommable des fruits séchés de Gola a été (5 à 6) fois supérieur à celui mesuré sur les jujubes des lots Sindia et Bandia. Au cours de la déshydratation, les fruits des lots d'origine sénégalaise prennent une coloration ocre clair, alors que les fruits de Gola deviennent très foncés, presque noirs.

\subsection{Caractéristiques biochimiques des fruits}

\subsubsection{Teneur en eau}

La partie comestible des jujubes africains a eu une teneur en eau comprise entre (59 et 68) \% de mf (tableau II). Cette concentration est proche des valeurs trouvées lors des travaux antérieurs $[18,19]$. Les fruits de Gola ont été plus riches en eau (84\%). Le séchage a abaissé les teneurs en eau à 20-23\% de mf, ce qui, selon Favier et al. [20], correspond à un état intermédiaire entre fruits secs et demi-secs.

\subsubsection{Teneur en composés organiques}

Les jujubes frais analysés ont eu une teneur en protéines comprise entre $(1,7$ et 3,7$) \%$ de mf et $(7,4$ et 11,8$) \%$ de ms (tableau II). Ces résultats sont du même ordre de 
Tableau I.

Quelques caractéristiques biométriques des fruits de trois lots de jujubes d'origine différente récoltés au Sénégal : les lots Bandia et Sindia sont issus de jujubiers locaux, le lot Gola correspond à une variété introduite (chaque valeur représente la moyenne de six mesures faites chacune sur un échantillon distinct de fruits prélevés dans le lot de jujubes considéré).

(a) Fruits frais (fruits de couleur jaune)

$\begin{array}{lcccccc}\begin{array}{l}\text { Variété } \\ \text { étudiée }\end{array} & \begin{array}{c}(\mathrm{A}) \\ \text { Poids d'un fruit } \\ (\mathrm{g})\end{array} & \begin{array}{c}(\mathrm{B}) \\ \begin{array}{c}\text { Poids de la matière } \\ \text { comestible d'un fruit } \\ (\mathrm{g})\end{array}\end{array} & \begin{array}{c}(\mathrm{C}) \\ \text { Volume d'un fruit } \\ \left(\mathrm{cm}^{3}\right)\end{array} & \begin{array}{c}(\mathrm{D}) \\ \begin{array}{c}\text { Volume de la matière } \\ \text { comestible d'un fruit } \\ \left(\mathrm{cm}^{3}\right)\end{array}\end{array} & \begin{array}{c}\text { Rapport } \\ {[\mathrm{B} / \mathrm{A}]}\end{array} & \begin{array}{c}\text { Rapport } \\ {[\mathrm{B} / \mathrm{A}]}\end{array} \\ \text { Gola } & 18,5 \mathrm{a} & 17,3 \mathrm{a} & 18,3 \mathrm{a} & 17,4 \mathrm{a} & 0,93 \mathrm{a} & 0,94 \mathrm{a} \\ \text { Bandia } & 2,1 \mathrm{~b} & 1,5 \mathrm{~b} & 2,1 \mathrm{~b} & 1,7 \mathrm{~b} & 0,73 \mathrm{~b} & 0,78 \mathrm{~b} \\ \text { Sindia } & 1,1 \mathrm{c} & 0,8 \mathrm{~b} & 1,1 \mathrm{c} & 0,9 \mathrm{~b} & 0,69 \mathrm{c} & 0,76 \mathrm{~b}\end{array}$

(b) Fruits séchés

\begin{tabular}{|c|c|c|c|c|}
\hline $\begin{array}{l}\text { Variété } \\
\text { étudiée }\end{array}$ & $\begin{array}{l}\text { (A) } \\
\text { Poids d'un fruit } \\
\text { (g) }\end{array}$ & $\begin{array}{l}\text { (B) } \\
\text { Poids de la matière } \\
\text { comestible d'un fruit } \\
\text { (g) }\end{array}$ & $\begin{array}{l}\text { Rapport } \\
\text { [B/A] }\end{array}$ & Couleur \\
\hline Gola & $3,7 \mathrm{a}$ & $2,6 \mathrm{a}$ & $0,73 \mathrm{a}$ & Brun-noir \\
\hline Bandia & $1,0 \mathrm{~b}$ & $0,5 \mathrm{~b}$ & $0,50 \mathrm{c}$ & Ocre \\
\hline Sindia & $0,8 \mathrm{~b}$ & $0,4 \mathrm{~b}$ & $0,56 \mathrm{~b}$ & Ocre \\
\hline
\end{tabular}

grandeur que ceux obtenus par Becker ou Favier et al. [7, 20]. Le séchage des fruits n’a pas modifié la teneur protéique des jujubes locaux. En revanche, les fruits de Gola ont perdu la moitié de leurs protéines pendant cette phase, cette teneur passant de $10,7 \%$ de ms dans les fruits frais à $5,4 \% \mathrm{~ms}$ dans les fruits séchés.

Les concentrations en vitamines $C$ mesurées dans la partie consommable des jujubes frais des lots Sindia et Bandia ont été (4 à 8) fois supérieures à celles mesurées par Toury et al. ou Favier et al. [18-20] pour les jujubes sauvages. Elles confirment cependant les observations d'autres auteurs $[4,7]$ qui avaient mesuré des concentrations en acide ascorbique des jujubes comprises entre (300 et 500) $\mathrm{mg} \cdot 100 \mathrm{~g}^{-1}$. Les jujubes originaires de Sindia ont été moins riches en vitamine $C$ que ceux des lots Bandia et Gola. Pour cette variété importée, la teneur de $373 \mathrm{mg} \cdot 100 \mathrm{~g} \mathrm{~g}^{-1} \mathrm{mf}$ obtenue s'est révélée être environ doublée par rapport aux mesures faites en Inde [5, 10, 17]. Ces variations parfois importantes peuvent en partie s'expliquer par le fait que la teneur en vitamine $C$ dépend grandement de l'état de maturité à la récolte [21] et du type de conduite culturale appliqué aux arbres [22]. Lors du séchage, les jujubes du lot Gola ont perdu les quatre cinquièmes de leur concentration en vitamine $\mathrm{C}$, alors que les jujubes du lot Bandia n'en ont perdu qu'un tiers et qu'aucune perte significative n'a été enregistrée pour le lot Sindia. Cette différence peut en partie s'expliquer par le séchage plus long des jujubes Gola.

Que les fruits soient frais ou séchés, les teneurs en sucres réducteurs des fruits des deux lots de jujubes sénégalais, rapportés à la masse de matière fraîche ou de matière sèche, ont été identiques (tableau II). Pour 


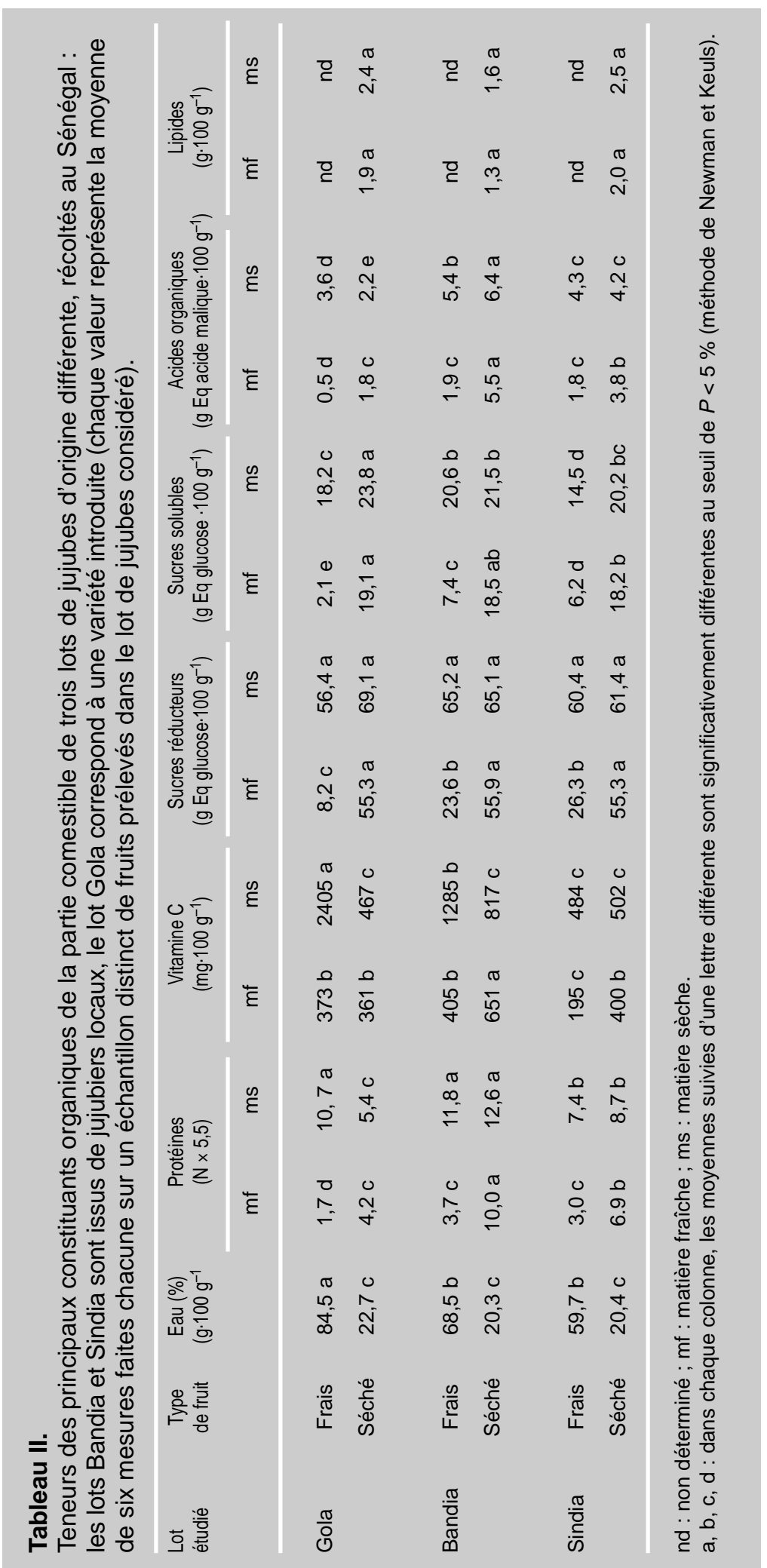


ces mêmes lots, les teneurs en sucres solubles ont été proches, même si les différences enregistrées pour les fruits frais ont été significatives. En revanche, les jujubes frais de la variété Gola ont été environ trois fois moins riches en sucres que les fruits des lots de provenance locale, différence pouvant s'expliquer, du moins partiellement, par une plus forte teneur en eau des fruits de la variété indienne. Les données bibliographiques portent essentiellement sur la concentration en sucres totaux, évaluée à des taux de (11 à 16) \% de mf pour les variétés indiennes, de (18 à 32) \% de $\mathrm{mf}$ pour les fruits frais des jujubiers sauvages et de (72 à 75) \% de mf pour les fruits séchés [5, 7, 8, 10, 18 20,22]. Notre analyse ayant distingué les sucres réducteurs et les sucres solubles, nous ne pouvons donc pas comparer directement ces valeurs données par la littérature avec nos résultats. Cependant, ils sembleraient être du même ordre de grandeur.

L'acidité des fruits, mesurée dans les lots Sindia et Bandia [de $(1,8$ à 1,9) g Eq acide malique pour $100 \mathrm{~g} \mathrm{mf}$ a été similaire, mais, rapportés à la masse de matière sèche, les résultats ont montré que les fruits du lot Sindia étaient moins acides. Quel que soit le type de fruits frais ou séchés et la référence prise ( $\mathrm{mf}$ ou $\mathrm{ms}$ ), la concentration en acides organiques des fruits du lot Gola [de $0,5 \mathrm{~g} \mathrm{Eq}$ acide malique pour $100 \mathrm{~g} \mathrm{mf}$ ] toujours été inférieure à celle des fruits locaux, quoique légèrement supérieure à celle mesurée en Inde : $(0,30$ à 0,35$) \mathrm{g}$ Eq acide malique pour $100 \mathrm{~g} \mathrm{mf}[10,17]$.

Les concentrations en lipides des fruits séchés ont été comprises entre $(1,3$ et 2,0) $\mathrm{g} \cdot 100 \mathrm{~g}^{-1} \mathrm{mf}$ et $(1,6$ et 2,5$) \mathrm{g} \cdot 100 \mathrm{~g}^{-1} \mathrm{~ms}$, sans différences significatives entre les trois lots de jujubes étudiés. Ces valeurs sont du même ordre de grandeur que les valeurs maximales estimées par Favier et al. [20].

\subsubsection{Teneur en éléments minéraux}

Les concentrations en calcium et en fer des jujubes locaux ont été du même ordre de grandeur que celles trouvées dans la littérature $[2,7,19,20]$ (tableau III). En revanche, nos résultats ont établi que la teneur en phosphore des fruits secs récoltés lors de notre expérimentation au Sénégal était double ou triple des valeurs données par Becker [7] ou Favier et al. [20].

Les teneurs en calcium, magnésium et fer, rapportées à la masse de matière sèche,

\section{Tableau III.}

Teneurs des principaux éléments minéraux $\left(\mathrm{mg} \cdot 100 \mathrm{~g}^{-1}\right)$ de la partie comestible de trois lots de jujubes d'origine différente, récoltés au Sénégal : les lots Bandia et Sindia sont issus de jujubiers locaux, le lot Gola correspond à une variété introduite (chaque valeur représente la moyenne de six mesures faites chacune sur un échantillon distinct de fruits prélevés dans le lot de jujubes considéré).

\begin{tabular}{|c|c|c|c|c|c|c|c|c|c|c|c|}
\hline \multirow{2}{*}{$\begin{array}{l}\text { Lot } \\
\text { étudié }\end{array}$} & \multirow{2}{*}{$\begin{array}{l}\text { Type } \\
\text { de fruit }\end{array}$} & \multicolumn{2}{|c|}{ Matière minérale } & \multicolumn{2}{|c|}{ Calcium } & \multicolumn{2}{|c|}{ Magnésium } & \multicolumn{2}{|c|}{ Phosphore } & \multicolumn{2}{|c|}{ Fer } \\
\hline & & $\mathrm{mf}$ & $\mathrm{ms}$ & $\mathrm{mf}$ & $\mathrm{ms}$ & $\mathrm{mf}$ & $\mathrm{ms}$ & $\mathrm{mf}$ & $\mathrm{ms}$ & $\mathrm{mf}$ & $\mathrm{ms}$ \\
\hline \multirow[t]{2}{*}{ Gola } & Frais & $1030 \mathrm{c}$ & $6660 \mathrm{a}$ & $76 \mathrm{~d}$ & $488 \mathrm{a}$ & $21 \mathrm{e}$ & $137 \mathrm{a}$ & $54 \mathrm{~b}$ & 403 a & $1,6 \mathrm{c}$ & $8,5 \mathrm{a}$ \\
\hline & Séché & $4625 a$ & $5980 \mathrm{a}$ & 204 b & $255 \mathrm{~b}$ & $62 \mathrm{~b}$ & $81 c$ & 207 a & $267 \mathrm{~b}$ & $2,2 \mathrm{bc}$ & $2,6 \mathrm{~b}$ \\
\hline \multirow[t]{2}{*}{ Bandia } & Frais & $1910 \mathrm{~b}$ & $6065 \mathrm{a}$ & $145 c$ & $460 \mathrm{a}$ & $36 \mathrm{~d}$ & $114 b$ & $77 \mathrm{~b}$ & $258 b$ & $1,5 \mathrm{c}$ & $4,9 \mathrm{~b}$ \\
\hline & Séché & 4450 a & $5580 \mathrm{a}$ & $380 \mathrm{a}$ & $477 \mathrm{a}$ & $88 \mathrm{a}$ & $109 \mathrm{~b}$ & 209 a & $262 b$ & $2,9 a$ & $3,6 \mathrm{~b}$ \\
\hline \multirow[t]{2}{*}{ Sindia } & Frais & $2200 \mathrm{~b}$ & $5460 \mathrm{a}$ & $169 \mathrm{bc}$ & $418 a$ & $45 c$ & $111 \mathrm{~b}$ & $59 \mathrm{~b}$ & $149 \mathrm{c}$ & $1,7 \mathrm{c}$ & $4,1 \mathrm{~b}$ \\
\hline & Séché & $4470 \mathrm{a}$ & $5650 \mathrm{a}$ & $379 a$ & 475 a & $87 a$ & $108 \mathrm{~b}$ & $181 \mathrm{a}$ & $248 b$ & $2,4 \mathrm{~b}$ & $3,0 \mathrm{~b}$ \\
\hline
\end{tabular}

$\mathrm{mf}:$ matière fraîche $; \mathrm{ms}:$ matière sèche.

$a, b, c, d$ : dans chaque colonne, les moyennes suivies d'une lettre différente sont significativement différentes au seuil de $P<5 \%$ (méthode de Newman et Keuls). 
ont été égales ou peu différentes pour les deux lots de jujubes locaux, que les fruits soient frais ou séchés. Elles n'ont pas présenté de perte lors du séchage. Les teneurs en magnésium, phosphore et fer des fruits de la variété Gola ont été supérieures à celles mesurées dans les fruits de variétés locales. Mais, au cours du séchage des fruits, il y a eu une diminution systématique des concentrations en éléments minéraux dans les jujubes de la variété Gola : diminution d'un tiers pour le phosphore, de moitié pour le calcium et le magnésium, des deux tiers pour le fer.

Rapporté à la matière fraîche, la portion comestible (fruits frais ou séchés) des fruits de la variété Gola a toujours été plus pauvre en calcium et magnésium que la portion comestible des fruits des lots Sindia et Bandia. Ces observations pourraient être expliquées d'une part par la plus grandeur teneur en eau des fruits frais de la variété Gola, d'autre part, par la perte d'une fraction importante de ces deux éléments pendant le séchage. En revanche, les concentrations en fer et en phosphore ont été proches pour les trois lots.

\subsection{Enquête organoleptique}

Le test gustatif réalisé auprès d'un panel de la population sénégalaise consommatrice traditionnelle de jujubes (figure 1) a montré que les fruits séchés sont, d'une façon générale, plus appréciés que les jujubes frais. Lorsqu'ils sont proposés en fruits frais, les jujubes des trois lots ont recueilli des notes proches (entre 3,4 et 3,6). Le produit préféré a été le jujube Gola séché, estimé comme bon à très bon, avec une note moyenne de 4,4 sur 5 . De nombreux dégustateurs ont trouvé que ce produit avait beaucoup plus d'arômes que les fruits des deux autres lots et ils l'ont comparé à la datte, confirmant ainsi certaines observations faites en Inde et en Amérique où ce produit est commercialisé sous le nom de Chinese dates [6].

\section{Conclusions}

Les fruits de Z. mauritiana Lam. ont des teneurs en sucres très élevées par rapport

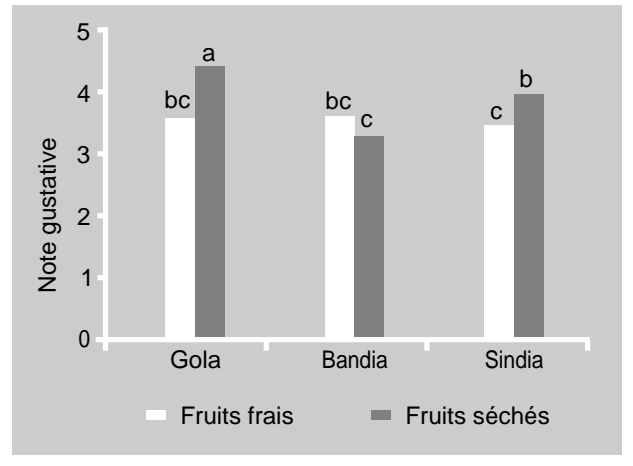

à d'autres fruits des zones de savanes africaines [13, 20]. Par ailleurs, leur teneur en vitamine $C$ est élevée comparativement à celle de nombreux fruits (datte, orange, citron), ce qui permet de classer les jujubes parmi les fruits les plus riches des zones sèches d'Afrique, au même niveau que la pulpe des fruits du baobab (Adansonia digitata L), de l'anacardier (Anacardium occidentale L.), de Parkia biglobosa (Jacq.) Benth. ou de Sclerocarya birrea (A. Rich.) Hochst. [7, 13, 19-21]. Le jujube est cependant moins riche que le ditakh (Detarium senegalense Gmelin) dont la teneur en vitamine C est estimée à $1290 \mathrm{mg}$ acide ascorbique. $100 \mathrm{~g}^{-1} \mathrm{mf}[18,19]$. Pendantle séchage des jujubes des variétés locales, les pertes en vitamine $\mathrm{C}$ sont faibles. En revanche, elles sont importantes lors du séchage des fruits du jujubier Gola, cela dans les mêmes proportions que celles mesurées après cuisson de certains légumes [23]. Cependant, en se basant sur la nécessité d'un apport quotidien en vitamine $C$ de (25 à 75) mg pour un adulte [24, 25], la consommation de (15 à 50) g de fruits séchés permettrait de couvrir la totalité des besoins journaliers.

La concentration en fer mesurée dans les jujubes est du même ordre de grandeur que celle estimée pour divers fruits (datte, banane, figue). Seule la pulpe du pain de singe (fruit du baobab) a une concentration double $[9,20]$. Cependant, certains légumesfeuilles d'Afrique présentent des concentrations en fer plusieurs dizaines de fois supérieures à celles observées pour ces fruits ; c'est le cas des feuilles de baobab ou de bissap (la roselle ou oseille de Guinée, Hibiscus sabdariffa L.), par exemple [26]. La consommation de $100 \mathrm{~g}$ de pulpe (soit une quarantaine de fruits séchés de Gola) couvrirait le quart des apports journaliers recommandés [25].
Figure 1.

Évaluation de la qualité gustative des fruits de trois lots de jujubes d'origine différente récoltés au Sénégal : les lots Bandia et Sindia sont issus de jujubiers locaux, le lot Gola correspond à une variété introduite. Les notes sont échelonnées de 0 , fruits les moins appréciés, à 5 , fruits les meilleurs. Les valeurs portant la même lettre appartiennent à un même groupe homogène (méthode de Newman et Keuls au seuil $P<5 \%$ ). 
Les concentrations en magnésium des jujubes sont faibles, comparées à celles mesurées dans de nombreux autres fruits sauvages [13, 26]. Leur teneur en phosphore est moyenne et bien inférieure à celle de certaines graines (baobab ou rocouyer, Bixa orellana L.) dont la teneur dépasse $400 \mathrm{mg}$ P. $100 \mathrm{~g}^{-1} \mathrm{mf}[18,27]$. En revanche, les jujubes seraient parmi les fruits des zones sèches les plus riches en calcium [13, $19,20]$, les feuilles de baobab, particulièrement riches, constituant une exception avec $2600 \mathrm{mg}$ Ca.100 g $\mathrm{g}^{-1} \mathrm{mf}$ [7]. Une consommation de $100 \mathrm{~g}$ de pulpe de jujubes secs couvrirait à peu près les besoins journaliers d'un adulte ou d'un enfant [25].

Nos résultats ont mis de plus en évidence que les fruits frais de la variété Gola étaient plus riches en eau que les jujubes locaux. Cette particularité peut expliquer la faiblesse relative des teneurs en éléments organiques et minéraux mesurées dans cette variété par rapport à celles caractérisant les fruits sauvages. Cette hypothèse est d'ailleurs renforcée par le fait que ces différences de concentration s'estompent, disparaissent même, après séchage des fruits ou lorsque les résultats sont rapportés à la masse de matière sèche. Cependant, (i) la teneur en protéines des jujubes secs de la variété Gola reste significativement inférieure à celle des fruits des deux lots sénégalais et (ii), pendant le séchage, les fruits de Gola perdent une proportion beaucoup plus importante de vitamine $C$ et d'éléments minéraux que les jujubes locaux. Ces constats, associés à la coloration noire prise par les jujubes Gola pendant le séchage, permettent d'émettre l'hypothèse d'un brunissement non enzymatique (réactions de Maillard) qui impliquerait les composés carbonylés (sucres réducteurs ou acide ascorbique) et l'azote aminé en présence d'eau [24]. Ces réactions de Maillard s'accompagnent généralement de la formation de différentes substances aromatiques [24], ce qui a bien été constaté lors des tests organoleptiques : les dégustateurs ont trouvé que les jujubes Gola séchés étaient très parfumés. Ce développement des arômes pourrait aussi être renforcé par une moindre acidité des fruits de cette variété.

Notre étude a donc précisé les caractères biométriques et la composition de fruits de
Z. mauritiana provenant de différentes origines. Elle a montré que les jujubes peuvent apporter de nombreux éléments - sucres, vitamine $C$, calcium et fer - importants pour la nutrition humaine. Ces fruits peuvent donc jouer un rôle significatif dans l'équilibre alimentaire des populations sahéliennes, d'autant qu'ils sont essentiellement consommés par les enfants [8] et que leur stockage à long terme est possible. Les qualités nutritionnelles de la variété Gola, en cours d'introduction au Sénégal, ne sont pas très différentes de celles des jujubes récoltés localement, surtout en ce qui concerne les fruits séchés qui constituent le mode principal de consommation des jujubes $[3,4,7,8]$. De plus, leurs propriétés organoleptiques semblent supérieures.

Divers travaux ont déjà démontré la faisabilité de la culture des variétés indiennes améliorées au Sahel [5, 12]. Ce travail démontre que les fruits produits correspondent au goût des populations et peuvent couvrir une part de leurs besoins nutritionnels. Ce constat conforte l'intérêt de développer la culture du jujubier au Sénégal et en Afrique de l'Ouest sous réserve que la rentabilité économique de cette culture et de sa transformation (séchage) soit prouvée. Des essais dans ce sens sont en cours.

\section{Remerciements}

Les auteurs remercient le personnel technique du Centre national de la Recherche forestière (Isra/CNRF), du laboratoire d'Analyses et d'essais (LAE) et du laboratoire de Microbiologie appliquée et de génie industriel (LMAGI) pour leur appui technique. Les premiers plants de la variété Gola ont été fournis par le programme IPALAC (International Program for Arid Land Crop, Israël).

\section{Références}

[1] Chevalier A., Les jujubiers ou Ziziphus de l'Ancien Monde et l'utilisation de leurs fruits, Rev. Bot. Appl. 27 (1947) 470-483. 
[2] Booth F.E.M., Wickens G.E., Non-timber uses of selected arid zone trees and shrubs in Africa, FAO Conserv. Guide 19, FAO, Rome, Italy, 1988.

[3] Baumer M., Arbres, arbustes et arbrisseaux nourriciers en Afrique occidentale, ENDA Éditions, Dakar, Sénégal, 1995.

[4] Munier P., Le jujubier et sa culture, Fruits 28 (1973) 377-388.

[5] Vashishtha B.B., Ziziphus for drylands - a perennial crop solving perennials problems, Agroforest. Today 9 (1997) 10-12.

[6] Prasad R.N., Bankar G.J., Vashishtha B.B., Products from ber fruits, Sci. Hortic. 5 (1995) 47-49.

[7] Becker B., The contribution of wild plants to human nutrition in the Ferlo (Northern Senegal), Agroforest. Syst. 1 (1983) 257-267.

[8] Bergeret A., Ribot J.C., L'arbre nourricier en pays sahélien, Éditions de la Maison des Sciences de l'Homme, Paris, France, 1990.

[9] Vashishtha B.B., Pareek O.P., Identification key for the cultivars of Indian jujube (Ziziphus mauritiana Lamk.), Ind. J. Hortic. 46 (1989) 183-188.

[10] Chovatia R.S., Patel D.S., Patel G.V., Performance of ber (Ziziphus mauritiana Lamk.) cultivars under arid conditions, Ann. Arid Zone 32 (1993) 215-217.

[11] Danthu P., Bâ A., Diallo I., Delhove G., Coly E.V., Sall P., Domestication and improvement of jujube tree (Zizyphus mauritiana) - State of art in Senegal, in: IUFRO (Ed.), Proc. XXI IUFRO World Congr., Vol. III, Kuala Lumpur, Malaisie, 2000, pp. 271-272.

[12] Danthu P., Soloviev P., Touré M.A., Gaye A., Propagation végétative d'une variété améliorée de jujubier introduite au Sénégal, Bois For. Trop. 272 (2002) 93-96.

[13] Tchiégang C., Dandjouma A., Dzudie T., Caractérisations physico-chimiques de cinq espèces fruitières endémiques de la savane camerounaise, Fruits 54 (1999) 413-422.

[14] Bremmer J.M., Mulvaney C.S., Nitrogen total, in: Am. Soc. Agron. (Ed.), Methods of soil analysis, Agron. Ser., Vol. 9, Part 2, Chemical and microbiological properties, Madison, Wisconsin, 1982, pp. 595-624.

[15] Miller G.L., Use of dinitrosalicylic acid reagent for determination of reducing sugar, Anal. Chem. 31 (1959) 426-428.
[16] Dubois M., Gillet K.A., Dosage des sucres totaux à l'ortho-toluidine, J. Agr. Food Chem. 13 (1956) 137.

[17] Chovatia R.S., Patel D.S., Patel G.V., Pate A.T., Pruning studies in ber (Zizyphus mauritiana Lamk.) under dryland conditions, Ann. Arid Zone 30 (1991) 353-356.

[18] Toury J., Lunven P., Giorgi R., Aliments de cueillette et de complément au Sénégal et en zone sahélienne, Qual. Plant. Mater. Veg. 8 (1961) 139-156.

[19] Toury J., Giorgi R., Favier J.C., Savina J.F., Aliments de l'Ouest africain. Tables de composition, Ann. Nutr. Aliment. 27 (1967) 73-127.

[20] Favier J.C, Ireland-Ripert J., Laussucq C., Feinberg M., Répertoire général des aliments. Tome 3. Table de composition des fruits exotiques, fruits de cueillette d'Afrique, Tec \& Doc Lavoisier, Inra, Orstom, Paris, France, 1993.

[21] Eromosele I.C., Eromosele C.O., Kuzhkuzha D.M., Evaluation of mineral elements and ascorbic acid contents in fruits of some wild plants, Plant Food. Hum. Nutr. 41 (1991) 151-154.

[22] Lal H., Prasad A., Pruning in ber (Zizyphus mauritiana Lamk.). III. Effect on yield and fruit quality, Punjab Hortic. J. 20 (1980) 162-166.

[23] Trémolières J., Serville Y., Jacquot R., Manuel élémentaire d'alimentation humaine. Tome 2. Les aliments, Les Éditions ESF, Paris, France, 1977.

[24] Frénot M., Vierling E., Biochimie des aliments Diététique du sujet bien portant, Collect. Biosciences et Techniques, Douin Éditeurs, Vélizy, France, 1997.

[25] Latham M.C., Human nutrition in the developing world, FAO Food Nutr. Ser. 29, FAO, Rome, Italie, 1997.

[26] Sena L.P., Vanderjagt D.J., Rivera C., Tsin A.T.C., Muhamadu I., Mahamadou O., Millson M., Pastuszyn A., Glew R.H., Analysis of nutritional components of eight famine foods of the Republic of Niger, Plant Food. Hum. Nutr. 52 (1998) 17-30.

[27] Glew R.H., Vanderjagt D.J., Lockett C., Grivetti L.E., Smith G.C., Pastuszyn A., Millson M., Amino acid, fatty acid and mineral composition of 24 indigenous plants of Burkina Faso, J. Food Compos. Anal. 10 (1997) 205-217. 


\section{Caracteres fisicoquímicos y organolépticos comparados de los frutos de azufaifos silvestres y los de la variedad Gola introducida en Senegal.}

Resumen - Introducción. Zizyphus mauritiana Lam. es un árbol frutal silvestre muy común en el Sahel. Sus frutos, las azufaifas, tradicionalmente explotadas mediante recolección en los rodales naturales, representan una importante fuente de ingresos para numerosas familias rurales. Se consumen frescos o secos. En Senegal, se está realizando desde hace algunos años un programa de introducción de azufaifos mejorados de origen indio. El objetivo de este estudio era comparar la primera variedad introducida (Gola) con dos lotes de azufaifas locales. Material y métodos. La comparación entre los frutos de diferente origen apuntó a las características biométricas de los frutos, a los contenidos de la porción comestibles en diversos compuestos orgánicos y en elementos minerales. El estudio se completó con una encuesta gustativa entre los consumidores. Resultados y discusión. La porción comestible de frutos frescos de Gola es unas veinte veces más importante (en peso y volumen) que la de las azufaifas locales. La pulpa de Gola es más rica en agua y menos concentrada en elementos orgánicos (azúcares, proteínas) y minerales (calcio, magnesio). Sin embargo, tras secado, los contenidos ya no presentan diferencias significativas, exceptuando las proteínas y algunos elementos minerales cuya riqueza en Gola disminuye sensiblemente durante el secado. Los frutos de Gola secados, muy perfumados, fueron los más apreciados por la población senegalesa. Conclusión. Sea cual sea su origen, las azufaifas son ricas en azúcares, vitamina C, calcio y hierro. Estos frutos representan un aporte nutricional importante para las poblaciones de las regiones áridas. La variedad Gola responde bien al gusto del consumidor senegalés. Se puede considerar su difusión en la zona saheliense siempre y cuando se resuelvan antes los problemas técnicos ligados a la producción de plantas y tras verificar la rentabilidad económica de este nuevo cultivo.

Senegal / Sahel / Zizyphus mauritiana / azufaifo / frutas / composición química / consumo de alimentos / domesticación 